\title{
Study on Reliability Allocation of Machinery System
} Miaomiao Zhang ${ }^{1, a^{*}}$, Yan Cao ${ }^{2, b}$, Zhou Fang $^{3, c}$ and Bei Chen ${ }^{4, d}$

${ }^{1}$ Department of Mechanical and Electronic Engineering, Xi'an Technological University, Xi'an, China

a654662115@qq.com,bjantonyz@163, c27381788@qq.com, d651723489@qq.com

Keywords: Machinery system; Distribution of failure rate prediction; Reliability allocation; Allocation model

\begin{abstract}
The reliability allocation of machinery system is studied on. The basic principle and criterion of reliability allocation are expounded in detail. The mathematical model of reliability allocation of machinery system is established according to distribution method of failure rate prediction. The reasonable distribution of the reliability allocation of machinery system is analyzed by concrete example data. It will have positive significance for the design and improvement of machinery system.
\end{abstract}

\section{Introduction}

The low reliability level and service life are always the two biggest obstacles restricting the products export of construction machinery in our country, especially for loaders. Due to the insufficient emphasis on research of reliability of machinery in the past, the failure data is not collected and analyzed. So the machinery that developed and produced in China is usually has short service life and low reliability. And therefore, the reliability analysis is especially important for machinery system. The reliability allocation is the key link for system reliability design. Its object is to make full use of the limited resources. The reliability allocation is to distribute system reliability indexes, which are presented by design program, to several subsystem or equipments or components rationally ${ }^{[4]}$.And then, the reliability target value of subsystem or components will be defined.

\section{Criterion of System Reliability Distribution}

System reliability distribution is to solve the following fundamental inequalities Structure

$$
\begin{aligned}
& R_{s}\left(R_{1}, R_{2}, \ldots R_{i}, \ldots R_{n}\right) \geq R_{s}^{*} \\
& \overrightarrow{g_{s}}\left(R_{1}, R_{2}, \ldots R_{i}, \ldots R_{n}\right)<{\overrightarrow{g_{s}}}^{*}
\end{aligned}
$$

During the above formulas,

$R_{s}^{*}$ _ The reliability target value of the system.

$\vec{g}$

$g_{s}$ _ The comprehensive constraint condition, including expenses, weights, volume, power dissipation, and so on. It is a vector function.

$R_{i}$ - The reliability target value of the $(\mathrm{N})$ unit

For a simple series-system, the upper formulas can be transformed to the following formula

$$
R_{1}(t) R_{2}(t) \ldots R_{i}(t) \ldots R_{n}(t) \geq R_{s}^{*}(t)
$$

If there is not any constraint conditions, Eq.(1) and Eq.(3) will have innumerable solutions. Even if it has constraint conditions, it may also have innumerable solutions. Therefore, the key to reliability allocation lies in the determination of a method, through which we can get an optimal 
solution of reliability allocation values rationally. Considering the characteristics of the reliability, and to insure the rationality and feasibility, we usually choose the parameters such as failure rate and reliability degree for reliability allocation. Generally we should abide the following criterions [5].

For the products, which are operating in harsh environment, we allocate them relative high reliability indexes, because for these products, the harsh environment may increase the failure rate of them. So only if they are allocated higher reliability indexes, they can work normally.

For the products, which are easy to maintenance and repair, we allocate them relative low reliability indexes, because for these products, they are easy to maintenance and repair even if they are breakdown, and therefore the system reliability can be insured.

For the units, which have high improving potential, we allocate them relative low reliability indexes, because it is much easier for these products to improve their reliability.

In addition, the allocation should be combined with actual situation, and consider other factors, such as allocating relative high reliability indexes to poor reliability products to obtain comprehensive effectiveness. For the goods-shelf-products and the developed systems with already existing reliability indexes, we should not carry on reliability allocation. Meanwhile, when carrying on reliability allocation, we should reject the reliability values of these units from overall performance.

\section{Mathematical Model of System Reliability Allocation}

Estimated Failure Rate Distribution Method. This method is suitable for the cascaded subsystem with a constant failure rate. The assigning procedures are as follow.

Set a goal—selecting $\lambda_{\mathrm{i}}$ according to the following formula

$$
\sum_{i=1}^{n} \lambda_{i}^{*} \leq \lambda_{s}
$$

During the above formula,

$\lambda_{i}^{*}$ __ Failure rate that allocated to the i subsystem before failure.

$\lambda_{\mathrm{g}} \longrightarrow$ Required system failure rate

Certain the subsystem failure rate $\lambda_{\mathrm{i}}$ according to historical data or prediction.

Certain the weighting factors $W_{\mathrm{i}}$ for each subsystem according to the failure rate determined by Eq. 2 as follow

$$
W_{i}=\frac{\lambda_{i}}{\sum_{i=1}^{n} \lambda_{i}}
$$

The required failure rate that allocated to the i subsystem is as follow

$$
\lambda_{i}^{*}=W_{i} \lambda_{s}
$$

\section{The Rational Distribution of Machinery System Reliability}

By mathematical model of machinery system, we can conclude that there are series models between each subsystem of machinery. In this paper, we use estimated failure rate distribution method to allocate system reliability ${ }^{[8]}$.

Take the loaders for example. According to a group of existing data provided by Ministry of Machinery Industry, the mean free error time (MFET) of whole machine and its subsystems are listed in Table 1. 
Table 1 The Mean Free Error Time (MFET) of Whole Machine and its Subsystems

\begin{tabular}{|c|c|c|c|c|c|}
\hline Name & The Engine & $\begin{array}{c}\text { Transmission } \\
\text { System }\end{array}$ & $\begin{array}{c}\text { Hydraulic } \\
\text { System }\end{array}$ & $\begin{array}{c}\text { Working } \\
\text { Device }\end{array}$ & $\begin{array}{c}\text { Other } \\
\text { Mechanism }\end{array}$ \\
\hline MFET(h) & 400 & 500 & 350 & 500 & 600 \\
\hline
\end{tabular}

Based on the industrial status in our country and the data of mean free error time (MFET) of some famous brand loaders in America and Japan, which is around 500 to 800 hours, we can confirm that the relatively advanced mean free error time of a whole machine is 550 hours. Owing to the reliability range is about $0.90 \sim 0.99$ that permitted in construction machinery products, and considering the reliability level of machinery in our country, we set $=0.9$. The loaders system reliability distribution obeys the Waybill distribution. After initial running-in period (about 750 900 hours), the failure rate tended to be stable, and obeys exponential distribution.

(1) Solving the system working time according to the required system reliability

$$
R_{s}=e^{-\lambda_{s} \times t_{s}}
$$

We can get the system working time: $t_{s}=-\ln R_{s} \times M_{s}=-\ln 0.9 \times 550=57.948 h$.

We can also get

$$
\lambda_{s}=\frac{1}{M_{s}}=1.8 \times 10^{-3} / \mathrm{h}
$$

by $\mathrm{M}_{s}$ in the system.

(2) Determine the failure rate of subsystem according to the mean free error time (MFET) of each subsystem

$$
\begin{gathered}
\lambda=\frac{1}{\text { The Mean Free Error Time (MFET) }} \\
\Rightarrow \lambda_{1}=2.5 \times 10^{-3} / \mathrm{h} ; \quad \lambda_{2}=\lambda_{4}=2 \times 10^{-3} / \mathrm{h} \\
\lambda_{3}=2.857 \times 10^{-3} / \mathrm{h} ; \lambda_{5}=1.667 \times 10^{-3} / \mathrm{h}
\end{gathered}
$$

(3) Based on Eq.5, we specify weighting factors for each subsystem

$$
\begin{aligned}
\Rightarrow W_{1} & =0.2268 ; & W_{2}=W_{4}=0.1814 \\
W_{3} & =0.2592 ; & W_{5}=0.1512
\end{aligned}
$$

(4) Calculate the failure rate that allocated to each subsystem according to Eq.6

$$
\begin{aligned}
\Rightarrow \lambda^{*}{ }_{1} & =0.412 \times 10^{-3} / h ; & \lambda^{*}{ }_{2} & =\lambda^{*}{ }_{4}=0.33 \times 10^{-3} / h \\
\lambda^{*}{ }_{3} & =0.471 \times 10^{-3} / h ; & \lambda^{*}{ }_{5} & =0.275 \times 10^{-3} / h
\end{aligned}
$$

(5) The degree of reliability that allocated to each unit correspondingly is

$$
\begin{aligned}
& R_{i}^{*}(t)=e^{-\lambda_{i}^{*} t} \\
& \Rightarrow R_{1}^{*}=0.976 ; \quad R_{2}^{*}=R_{4}^{*}=0.982 \\
& \quad R_{3}^{*}=0.973 \quad R_{5}^{*}=0.984 \\
& R_{s}^{*}(57.948)=R_{1}^{*} \cdot R_{2}^{*} \cdot R_{3}^{*} \cdot R_{4}^{*} \cdot R_{5}^{*}=0.9011>R_{s}
\end{aligned}
$$

\section{Conclusion}

Understanding and mastering reliability characteristics of machinery system could provide important basis to manufacturer for developing and optimizing the machinery system and its corresponding built-up parts. But for current domestic status of machinery, manufacturers rarely carry research and analysis of reliability on machinery system. The reasons are as follow: machinery is usually large complex system, with very few test samples and malfunctions data. It is hard to accurate quantify and calculate. In this paper we have studied on the reliability allocation of machinery system using estimated failure rate distribution method. Through bench reliability test of whole machine and components, we can obtain the correlated reliability data. On the basis of 
reliability allocation results, we can optimize the component design to improve the main frame reliability, so as to improve and enhance the products quality constantly. It will offer a reference and basis to reliability engineering popularization in machinery industry, and therefore achieve an advanced reliability management and improve the working properties of machinery.

\section{Acknowledgements}

The paper is supported by Key Problem Tackling Project of Shaanxi Scientific and Technological Office (2016GY-024).

\section{References}

[1] Z. W. Cong, Method and Case Study Research on Production System Reliability Allocation. (Ph.D., Jilin University, China 2014), p37

[2] B.Y. Li, Study on FTA and AHP Based Reliability Allocation Method. (Ph.D., University of Science and Technology of China, China 2014), p8

[3] X.F. Hao, Research on Optimal Allocation Method of Reliability Allocation of Complicated System. (Ph.D., Northeastern University, China 2008), p26

[4] M.L He, Y.L. Guo, System Reliability Allocation Method Based on Importance Degree [J]. Safety and Environmental Engineering, Vol. 16 (2009) No.4, p63

[5] C. Dong, System Reliability Assignment Method [J]. Systems engineering and Electronics Distribution System, Vol.36 (1996) No.7, p37

[6] Y.F. Shao, H.J Xue, Y.G Zhang, A Reliability Allocation Method for Complicated Series-parallel Systems [J]. Aircraft Design, Vol. 27 (2007) No.2, p53

[7] M. Fang, Research on Reliability Allocation Method of Mechanical System in Conceptual Design Stage. (Ph.D., Tianjin University, China 2014), p15

[8] D.N. Li, M.Y. Zhang, C.Q. Ma, Allocation Method of Fuzzy Reliability for Parallel and Series Connection Systems [J]. Journal of Gansu Sciences, Vol. 20 (2008) No.3, p103

[9] H.Z. Huang. Fuzzy Method of Mechanical System Reliability Distribution [J]. Mechanical Science and Technology, Vol. 15 (1996) No.2, p185

[10] Y. Zhang, Y.Q Liu. Effective Method of System Reliability Allocation [J]. Journal of Shenyang Polytechnic University, Vol. 21 (1999) No.2, p164 